\title{
Pontius' Handwash
}

\section{Victor Lage de Araújo MD IFCAP MSc*}

Laboratory Medicine, Brazil, International Fellow, College of American Pathologist, MSc, Evidence-Based Healthcare (UCL)

*Corresponding Author: Victor Lage de Araújo, MD, IFCAP, MSc. E-mail: victor.araujo.15@ucl.ac.uk

Received: October 18, 2019; Published: November 01, 2019

DOI: $10.31080 /$ ASMI.2019.02.0420

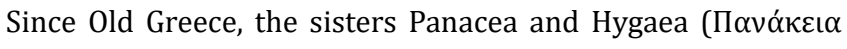
and $\left.\Upsilon \gamma \varepsilon \varepsilon^{\prime} \alpha\right)$ contend in vain for their father's attention. Each of

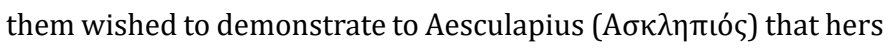
was the most effective way to handle human's diseases [1].

As a notable Doctor from our history, Ignatz Phillip Semmelweis [1818 -1865] [2] may have used the crafts of both sisters, but he favoured Hygaea. Semmelweis studied the incidence of Puerperal Fever in both wards of an obstetric clinic at Vienna; at that time, women would often deliver their babies while waiting for attention at the second unit (were only the Midwives cared for them) than to go to the doomed first unit (where Medical Students were the caregivers). Establishing what might have been the first documented Epidemiological Study [1944] (Encyclopaedia Britannica, 2019), the doctor observed that Puerperal Fever was two to three times higher at the physician's unit than that at the Midwives'. While people still discussed the Germ Theory of transmission of diseases [3] and contrasted it against the Miasma Theory, he hypothesised the students were carrying something invisible in their hands, from patients to dead bodies and backwards (only the students performed necropsies). He proposed that all physicians were to immerse their hands in a solution of carbolic acid before handling the patients. As a consequence, the infection rate on the Doctor's ward fell $18.27 \%$ to $1.27 \%$ - much below that of the Midwives' section. The fact was: hand-washing was undoubtedly not a generalised habit.

While most historical sources claim Semmelweis had some psychiatric ailment and died some years later, others say he died fighting for Infection Control Practices. More romantic sources claim that he somehow infected himself with a scalpel and died of Sepsis - the same sickness against which he had been struggling.

By 1928, another notable scientist, Alexander Fleming (18811955) [4] was to follow Panacea's paths. Purportedly with the help an incidental fact (he had previously left a Petri dish unattended during extended time), he discovered a new promising substance: Penicillin, the first antibiotic and miracle wonder that inhibited the growth of bacteria in the vicinity of the place where some fungus had grown and may even nowadays be active against some bacteria.

Alas! As soon as 1940, the first resistant strains of Escherichia coli could be documented [4]. The Medical Community would soon proceed to use antimicrobials extensively; and as bacteria developed, a race for new antimicrobials has started [5].

Since then, human science and professions have evolved a lot. Many new medical specialities now exist, such as Epidemiologists, Researchers, Infectologists, and Pathologists. Not all of them often do autopsies or surgery. The profession of Infection Control Practitioner (ICP) is now an interdisciplinary team, involving Physicians, Nurses, Technologists, Statisticians and a lot of Health-related professionals. The Pharmaceutical Industry has developed and produced a myriad of new molecules - some of which we can use as effective antimicrobial drugs and disinfectants [5].

Some events, however, were unanticipated: We now have agile computers (often meaning physical contact with keyboard and mouse), tablets and smartphones (which we regularly touch and stack into our pockets, along with other items). All Industries try to catch up by developing suitable disinfection means and preventive strategies [6].

Meanwhile, some phenomena are still the same: Healthcare Professionals commonly touch the patients, environments and objects, unaware of the ubiquitous presence of what is even at this moment evolving into evil Multi-resistant organisms (MRO). Microorganisms are still able to grow at an astounding pace and develop resistance to the attacks we direct to them [7].

While Panacea and Hygaea are an infinite stalemate, of one thing we must be aware: That we must be much more Biblical than Pontius Pilate when we wash our hands $[8,9]$. Then - let us pray the Antibiotics' use can be refrained and dedicated to the severe infections, where and while they can be most useful (Figure 1). 


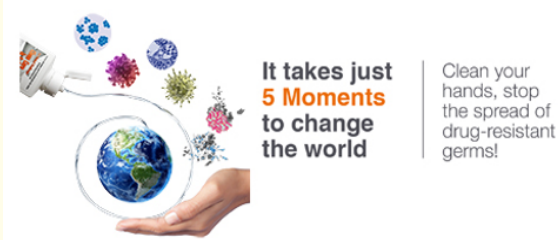

Figure1: The WHO. Hand-wash helps avoiding overuse of antibiotics and spread of drug-resistant germs. Available at https:// www.who.int/gpsc/5may/5may2014_top.jpg (17/10/2019).

\section{Bibliography}

1. Dye C Hygea and Panacea. Is prevention better than cure? (2006): 23

2. Encyclopaedia Britannica. Ignaz Semmelweis. German-Hungarian physician (2019).

3. Karamanou M., et al. "From miasmas to germs: a historical approach to theories of infectious disease transmission". Le Infezioni in Medicina 20.1 (2012): 58-62.

4. Lobanovska MM and Pilla G. "Penicillin's Discovery and Antibiotic Resistance: Lessons for the Future?". Yale Journal of Biology and Medicine 90 (2017):135-145.

5. Ventola CL. "The Antibiotic Resistance Crisis. Part 1: Causes and Threats". Pharmacy and Therapeutics 40.4 (2015).

6. Graveto JM., et al. "Cell Phone Usage by Health Personnel: Preventive Strategies to Decrease Risk of Cross Infection in Clinical Context". Texto Contexto Enferm 27.1 (2018): e5140016.

7. CDC. Biggest Threats and Data (2019).

8. Bible Hub. The Bible: Matthew 27 (2019): 24.

9. HEALIO Endocrine today. 2008. Penicillin: An accidental discovery changed the course of medicine (2008).

Volume 2 Issue 12 December 2019

(C) All rights are reserved by Victor Lage de Araújo. 